\title{
ОЦЕНКА ПСИХОЛОГИЧЕСКОГО БЛАГОПОЛУЧИЯ КАК ПРИНЯТИЕ РЕШЕНИЯ: ОСОБЕННОСТИ СУБЪЕКТИВНЫХ КРИТЕРИЕВ ПРИ ПСИХИЧЕСКИХ ЗАБОЛЕВАНИЯХ (НА МОДЕЛЯХ НЕПСИХОТИЧЕСКОЙ ДЕПРЕССИИ И ПЕРВОГО ПСИХОТИЧЕСКОГО ПРИСТУПА В ЮНОШЕСКОМ BO3PACTE)
}

\author{
Е.И. РАССКАЗОВА ${ }^{\mathrm{a}, \mathrm{b}, \mathrm{c}}$, С.Н. ЕНИКОЛОПОВ ${ }^{\mathrm{a}, \mathrm{b}}$, В.В. ГУЛЬДАН
}

${ }^{a}$ ФГБНУ «Научный центр психического здоровья», 115522, Россия, Москва, Каширское иоссе, д. 34 ${ }^{5}$ Московский государствениый университет имени М.В. Ломоносова, 119991, Россия, Москва, Ленинские горы, Ә. 1

'Национальный исследовательский университет «Высиая иккола әкономики», 101000, Россия, Москва, ул. Мясниикая, д. 20

${ }^{2}$ Московский областной щентр социальной и судебной психиатрии, 127083, Россия, Москва, ул. 8 Mapma, $\partial .1$

\section{Резіоме}

Оценка качества своей жизни, удовлетворенности и счастья рассматривается как результат принятия решения, субъективные критерии которого могут различаться как в норме, так и при психических заболеваниях. Понятия удовлетворенности жизнью, счастья и качества жизни понимаются как конкретные аспекты интегративного понятия психологического благополучия, предложенные в рамках разных научных традиций. В ходе анализа модерации на основе опросника качества жизни и удовлетворенности для психических заболеваний и шкалы счастья С. Любомирски сравнивается вклад качества жизни в разных сферах в общую оценку удовлетворенности жизнью и субъективное счастье в трех группах юношей 17-28 лет: больных непсихотическими депрессиями $\left(\mathrm{n}_{1}=76\right)$, больных в становлении ремиссии после первого психотического приступа $\left(\mathrm{n}_{2}=90\right)$, респондентов без психических заболеваний $\left(\mathrm{n}_{3}=185\right)$. Хотя все сферы в той или иной мере важны для общих оценок удовлетворенности и счастья, при непсихотических депрессиях здоровье, эмоциональная сфера и функционирование в течение дня более значимы для вывода о своей жизни по сравнению с двумя другими группами. Больные в становлении ремиссии после психотического приступа в оценках своего благополучия в наименьшей степени по сравнению с другими рес-

Работа выполнена при поддержке гранта Президента РФ для государственной поддержки молодых российских ученых, проект № МК4230.2015.6 «Отношение к болезни и качество жизни при психических заболеваниях». 
пондентами ориентируются на эмоциональную сферу, сферу общения и материальное благополучие. Результаты обсуждаются с позищий когнитивного подхода к пониманию и психотерапии при психических заболеваниях с акцентом на важность учета субъективных критериев и процесса оценки больными своего состояния и благополучия.

Ключевые слова: качество жизни, удовлетворенность, субъективное счастье, субъективные критерии оценки благополучия, непсихотическая депрессия, ремиссия после психотического приступа, юношеский возраст.

Со становлением биопсихосоциального подхода в медицине качество жизни стало одной из ключевых зависимых переменных психологического уровня анализа как в научных исследованиях, так и в практике (McDowel, 2006; Рассказова, Тхостов, 2015): призыв лечить не болезнь, а больного тесно связан с необходимостью учитывать его собственное ви́дение своего состояния, болезни и лечения. Однако до настоящего времени бо́льшая часть исследований ориентирована на понимание границ, структуры и предикторов качества жизни и психологического благополучия (Sirgy et al., 2006; Schalock, 2004; Ilic et al., 2010).

В психиатрии и клинической психологии немало внимания уделяется вопросу об особенностях качества жизни при различных заболеваниях, однако обсуждение ведется также в терминах более низкого/более высокого уровня благополучия. В частности, показано, что у психически здоровых испытуемых качество жизни и удовлетворенность связаны с уровнем депрессивности и обсессивнокомпульсивной симптоматики (Goracci et al., 2005, 2007). При шизофрении, шизоаффективных и аффективных расстройствах (Ritsner et al., 2005) они снижены, в том числе при депрессивных расстройствах даже в тех случаях, когда симптоматика минимальна (Nierenberg et al., 2010). Данные в отношении биполярных расстройств менее однозначны (Chand et al., 2004). У больных с аффективными расстройствами особенности качества жизни позволяли независимо от выраженности депрессивной и тревожной симптоматики предсказать длительность пребывания в больнице (Hope et al., 2009).

С нашей точки зрения, ключевой интерес для психологии представляют два других вопроса. Во-первых, субвективная оценка своей жизни (в терминах ее качества, удовлетворенности или сиастья) представляет собой процесс принятия решения, что делает актуальным вопрос о субъективных критериях и содержании этого процесса. На какие сферы своей жизни обращает внимание человек, оценивая свою жизнь? Являются ли эти сферы универсальными или они могут быть индивидуально специфическими? Могут ли низкие показатели качества жизни и субъективного благополучия, например при депрессиях, объясняться особенностями субъективных критериев оценки, когда одни из сфер становятся особенно значимыми, а другие - менее значимыми? Заметим, что представление о важности учета субъективных критериев оценки 
своего качества жизни высказывалось в отношении психосоматических заболеваний (Садальская, Ениколопов, 2001) и хорошо согласуется с представлениями, базирующимися на когнитивном подходе к психическим заболеваниям, об избирательном внимании к событиям в зависимости от доминирующих убеждений человека (Бек и др., 2003; Clark et al., 1999; Beck et al., 2009). Данная гипотеза имеет важное практическое следствие: например, если низкий уровень качества жизни при депрессиях определяется не столько аффективными симптомами, сколько их субъективной значимостью для больного, именно процессы оценки, а не симптомы должны находиться в центре внимания при психотерапевтической работе.

Во-вторых, какие функиии выполняет субъективная оценка своей жизни в регуляции дальнейщей жизнедеятельности человека? Приведем пример двух человек, не удовлетворенных своей жизнью, у одного из которых это вызывает апатию и бессилие, а у другого - активные усилия по изменению и улучшению своей жизни. Какие факторы определяют то, какую функцию выполняет оценка качества своей жизни и благополучия?

Данная работа направлена на эмпирическую проверку первого вопроса: выявления общих и специфических для различных психических заболеваний субъективных критериев оценки удовлетворенности своей жизнью и благополучия. Спектр определений качества жизни и благополучия настолько широк (см.: Рассказова, 2012а), что позволяет некоторым авторам говорить о «зонтике» благополучия - как о сходящихся очень разных определениях-«спицах», поэтому в данной работе поясняется операционализация понятий «качество жизни», «психологическое благополучие» и «удовлетворенность жизнью».

В соответствии с психологической традицией мы будем использовать понятие психологического (субъективного) благополучия как интегративное понятие (Diener, Ryan, 2009), объединяющее как результаты когнитивной оценки своей жизни (удовлетворенность жизнью), так и эмоциональные переживания. Конструкт субъективного счастья (Lyubomirsky, 2013; Аргайл, 2003) также используется в данной традиции с акцентом на его более холистическую и эмоциональную природу по сравнению с удовлетворенностью жизнью. В данной работе общая оценка субъективного благополучия рассматривается в двух проявлениях - удовлетворенности жизнью и субъективного счастья.

В медицинской традиции понятие качества жизни изначально было призвано установить не только субъективную оценку человеком своего состояния, но и ограничения в его жизни и функционировании, связанные с заболеванием (как субъективные, так и по возможности объективные). Это понятие развивалось от максимально объективизированной оценки (например, может ли респондент взбежать на два пролета лестницы вверх без одышки) к более субъективной (например, оценка своего здоровья по шкале); а также от предпочтения негативных индикаторов качества жизни (например, депрессии, стресса) к учету позитивных 
индикаторов (удовлетворенности, субъективного здоровья и т.П.). При этом особое внимание уделялось доменам или сферам качества жизни. В данной работе применяется субъективно-ориентированное доменное определение качества жизни как субъективной оценки своего состояния и удовлетворенности в различных сферах (McDowel, 2006).

Мы предполагаем, что качество жизни в различных сферах выступает основой для общей оценки своего благополучия - при психических заболеваниях одни из сфер становятся субъективно более значимыми критериями для оценки, другие менее значимыми, чем частично обусловлено изменение в уровне благополучия, характерное для этих заболеваний. Косвенно подтверждают это предположение исследования на основе индивидуализированных инструментов оценки качества жизни (Martin et al., 2007, Wettergren et al., 2009): удовлетворенность в наиболее значимых для каждого респондента сферах (которые он называет в начале обследования) тесно связана с его благополучием. Для диагностики качества жизни в различных сферах в данной работе используется краткая версия опросника качества жизни и удовлетворенности, разработанная для психических заболеваний (Ritsner et al., 2005) на основе качественного отбора тех сфер, которые наиболее важны для больных.

Целью данного исследования является выявление особенностей субъективных критериев оценки удовлетворенности жизнью и счастья в норме при непсихотических депрессиях и после первого психотического приступа в юношеском возрасте. В частности, рассматривался акцент при принятии решения об общей удовлетворенности жизнью и счастьем на таких жизненных сферах, как: здоровье, общение, эмоциональное состояние, активность в свободное время, лечение, функционирование в течение дня, материальное благополучие.

Выдвигались следующие zunomeзы:

1. Краткий опросник качества жизни и удовлетворенности в модификации для психических заболеваний является надежным и валидным инструментом оценки качества жизни при психических заболеваниях. Поскольку до настоящего времени апробация русскоязычной версии методики проводилась лишь в норме (Рассказова, 2012б), предварительный анализ данных был посвящен оценке надежности-согласованности шкал методики в двух клинических группах, проверке критериальной валидности (предполагалось, что наиболее низкий уровень качества жизни должен быть у больных с депрессиями, а наиболее высокий - у респондентов контрольной группы) и конвергентной валидности (предполагалось, что шкалы опросника качества жизни будут положительно коррелировать со шкалой субъективного счастья и отрицательно - с рядом шкал опросника выраженности психопатологической симптоматики).

2. Как в контрольной, так и в обеих клинических группах общие оценки удовлетворенности жизнью и счастья зависят от качества жизни в конкретных сферах: сферах здоровья, эмоций, активности в свободное время, общения, а также удовлетворенности 
отношениями с другими людьми, функционированием в течение дня, материальным положением, а в клинических группах - от удовлетворенности лекарствами.

3. Вклад качества жизни в разных сферах в общее благополучие различается: качество жизни в эмоциональной сфере и сфере общения связано с оценкой удовлетворенности жизнью и счастья сильнее, а удовлетворенность активностью в свободное время и благополучием - слабее. При этом, поскольку удовлетворенность жизнью рассматривается как скорее когнитивная оценка (Diener, Ryan, 2009), а субъективное счастье предполагает менее дифференцированную и более основанную на эмоциях оценку (Аргайл, 2003), предполагалось, что вклад качества жизни в разных сферах в удовлетворенность жизнью будет бо́льшим, нежели в уровень счастья.

4. Хотя субъективные критерии оценки своей жизни при психических заболеваниях те же, что и в норме, их значимость и, соответственно, вклад качества жизни в разных сферах в общую оценку меняются. Так, больные с депрессиями в оценке удовлетворенности жизнью в большей степени опираются на качество жизни в сфере здоровья и эмоциональной сфере, а также функционирование в течение дня, тогда как для больных в ремиссии после психотического приступа, напротив, эмоциональная сфера и качество общения с окружающими менее важны.

\section{Процедура и методы исследования}

В исследовании участвовали три группы испытуемых (всего 351 человек): две клинических и одна контрольная. Все испытуемые были юношеского возраста (17-28 лет) и мужского пола. Клинико-психологическая часть исследования проводилась в ФГБНУ «Научный центр психического здоровья», в отделе по изучению эндогенных психических расстройств и аффективных состояний ФГБНУ НЦПЗ (директор Т.П. Клюшник) $)^{1}$

С больными обеих клинических групп проводилось интервью с целями формирования мотивации к исследованию и выявления особенностей отношения к заболеванию и его влиянию на их жизнь. Затем респонденты заполняли батарею психологических опросников. В контрольной группе вместо интервью кратко рассказывалась общая цель исследования, а затем респонденты заполняли методическую батарею.

В первую клиническую группу вошли 76 пациентов с депрессивным синдромом в рамках следующих непсихотических расстройств: расстройства настроения (41.7\%, коды диагнозов по МКБ-10: F31.3, F31.4, F32, кроме F32.2, F33, кроме F33.3, F34); расстройства личности (27.8\%, код диагноза по МКБ-10: F60) или шизотипическое расстройство (30.6\%, код диагноза по МКБ-10: F21). Средний возраст в группе составил $20.2 \pm 2.8$ года.

${ }^{1}$ Авторы благодарны В.Г. Каледе, А.Н. Бархатовой и М.А. Омельченко за помощь в организации исследования и сбора данных. 
Во вторую клиническую группу вошли 90 больных на этапе становления ремиссии после первого психотического приступа в рамках приступообразной шизофрении. Первый приступ был выбран с целью минимизировать роль дефекта в оценке качества жизни пациентов. Критерием исключения были выраженные негативные симптомы. Средний возраст в группе составил $21.8 \pm 3.2$ года.

Контрольную группу составили 185 жителей Москвы и Московской области юношеского возраста мужского пола. Критериями исключения были психические заболевания, черепно-мозговые травмы в анамнезе, зависимости от психоактивных вешеств. Средний возраст в группе составил $20.3 \pm 2.9$ года.

В данное исследование вошли следующие опросники более общей методической батареи:

1. Шкала счастья С. Любомирски (Осин, Леонтьев, 2008; Lyubomirsky, Lepper, 1999) - скрининговая методика оценки уровня субъективного счастья, состоящая из 4 пунктов.

2. Краткий опросник качества жизни и удовлетворенности в модификации для психических заболеваний (Рассказова, 2012б; Ritsner et al., 2005) включает четыре основные шкалы, характеризующие качество жизни в сфере здоровья, эмоциональной сфере, сфере активности в свободное время и сфере общения.

3. Для оценки выраженности жалоб на психопатологические симптомы использовался Опросник психопатологической симптоматики (SCL90R) в адаптации Н.В. Тарабриной (2001). Опросник состоит из списка 90 симптомов, наличие и выражен- ность каждого из которых респондент оценивает по шкале Лайкерта от 0 до 4 баллов. Включает следующие шкалы: «Соматизация», «Обсессивность-компульсивность», «Межличностная тревожность», «Депрессивность», «Тревожность», «Враждебность», «Фобии», «Паранойяльность», «Психотизм»; а также три общих индекса: «Общий симптоматический индекс» представляет сумму всех ответов и характеризует как тяжесть, так и количество симптомов; «Индекс проявления симптоматики» отражает только количество неотрицательных ответов, но не тяжесть симптомов; «Индекс выраженности дистресса» является ключевым общим показателем и отражает среднюю субъективную тяжесть симптоматики. В данном исследовании методика применялась только в клинических группах с целью предварительной проверки внешней валидности опросника удовлетворенности и качества жизни.

Обработка данных проводилась в программе SPSS Statistics 17.0.

\section{Результаты}

Качество жизни в разньх сферах и субвективное счастье при непсихотических депрессиях и ремиссиях после первого психотического приcтупа: предварительньй анализ

Поскольку данные о надежности русскоязычных версий Краткого опросника качества жизни и удовлетворенности и Шкалы счастья относятся к психически здоровым людям, предварительной задачей было установление надежности-согласованности этих показателей при непсихотических депрессиях и ремиссиях 
после первого психотического приступа в юношеском возрасте, а также исследование внешней и критериальной валидности Краткого опросника качества жизни и удовлетворенности при применении к больным с психическими заболеваниями.

Во всех случаях показатели надежности-согласованности варьировали от приемлемых до хороших, что позволяет использовать эти показатели в клинико-психологических исследованиях (таблица 1).

В подтверждение критериальной валидности Краткого опросника качества жизни и удовлетворенности для психических заболеваний и Шкалы счастья как общий уровень субъективного счастья, так и качество жизни в разных сферах минимальны у больных с депрессиями и максимальны - в контрольной группе $(\mathrm{F}=15.50-54.84, \mathrm{df}=2, p<0.01)$. Согласно результатам попарного сравнения групп post hoc по критерию Шеффе, все группы значимо различаются по качеству жизни в сфере общения, эмоциональной сфере и сфере активности в свободное время $(p<0.01)$. Хотя больные, перенесшие первый приступ шизофрении, оценивают свой уровень счастья, удовлетворенность жизнью в целом и качество жизни в сфере здоровья несколько ниже, чем респонденты контрольной группы, эти различия не достигают принятого уровня значимости. Напротив, те же показатели у больных с депрессиями значимо снижены по сравнению с двумя другими группами $(p<0.05)$.

В подтверждение внешней (конвергентной) валидности Краткой версии опросника качества жизни и удовлетворенности для психических заболеваний в клинических группах качество жизни в сферах здоровья, эмоций, активности в свободное время и общения, а также общая удовлетворенность жизнью положительно коррелируют со шкалой счастья $(r=0.36-0.58, p<0.01$ при депрессиях и $r=0.29-0.47, p<0.01$ при становлении ремиссии после психотического приступа). Корреляционный анализ шкал Опросника

Таблица 1

Надежность-согласованность шкал оценки качества жизни в разных сферах

\begin{tabular}{|l|c|c|c|}
\hline \multirow{2}{*}{\multicolumn{1}{c|}{ Показатели }} & \multicolumn{3}{|c|}{ К Кронбаха } \\
\cline { 2 - 4 } & $\begin{array}{c}\text { Контрольная } \\
\text { группа }\end{array}$ & $\begin{array}{c}\text { Непсихотические } \\
\text { депрессии в } \\
\text { юношеском возрасте }\end{array}$ & $\begin{array}{c}\text { Ремиссия после } \\
\text { психотического } \\
\text { приступа }\end{array}$ \\
\hline КЖ в сфере здоровья & 0.77 & 0.83 & 0.73 \\
\hline КЖ в эмоциональной сфере & 0.75 & 0.76 & 0.80 \\
\hline $\begin{array}{l}\text { КЖ в сфере активности } \\
\text { в свободное время }\end{array}$ & 0.69 & 0.68 & 0.72 \\
\hline КЖ в сфере общения & 0.67 & 0.73 & 0.70 \\
\hline Удовлетворенность жизнью & 0.79 & 0.84 & 0.74 \\
\hline Субъективное счастье & 0.74 & 0.73 & 0.68 \\
\hline
\end{tabular}


выраженности психопатологической симптоматики позволяет сделать следующие выводы.

- Общая удовлетворенность жизнью при депрессиях отрицательно коррелирует с выраженностью обсессивно-компульсивных симптомов, межличностной тревоги, депрессии, фобической симптоматики и субпсихотических симптомов $(r=$ $=-0.37 \ldots-0.24, p<0.05)$. При этом качество жизни в эмоциональной сфере отрицательно коррелирует с выраженностью всех психопатологических симптомов $(r=-0.45 \ldots-0.27$, $p<0.05)$; качество жизни в сфере здоровья - с выраженностью симптомов соматизации, обсессивностикомпульсивности, депрессии, тревоги, фобической симптоматики ( $r=$ $=-0.36 \ldots-0.25, p<0.05)$. Качество жизни в сфере активности в свободное время отрицательно связано с симптомами соматизации, обсессивности-компульсивности, депрессии и фобии $(r=-0.36 \ldots-0.24, p<0.05)$, а качество жизни в сфере общения только с симптомами обсессивностикомпульсивности и депрессии ( $r=$ $=-0.31 \ldots-0.30, p<0.01)$.

- При становлении ремиссий после психотического приступа ни общая удовлетворенность жизнью, ни качество жизни в сфере общения не связаны с выраженностью психопатологической симптоматики. Напротив, качество жизни в сфере здоровья и эмоциональной сфере отрицательно коррелирует с большинством симптомов, кроме тревоги и фобий $(r=-0.45 \ldots-0.22, p<0.05)$. Качество жизни в сфере активности в свободное время отрицательно связано с симптомами соматизации, обсессивности-компульсивности, межличностной тревоги и депрессии $(r=-0.34 \ldots-0.27, p<0.05)$.

В целом эти данные согласуются с данными предыдущих исследований (Рассказова, 2012б; Goracci et al., 2005, 2007; Ritsner et al., 2005; Nierenberg et al., 2010) и подтверждают надежность, критериальную и внешнюю валидность опросника качества жизни и удовлетворенности в исследованиях больных с психическими заболеваниями. Однако ключевым для данного исследования был другой вопрос: могут ли низкие показатели качества жизни и субъективного благополучия, особенно характерные для больных с депрессиями, обьясняться тем, что они используют специфические субъективные критерии оценки себя и различных сфер своей жизни, например, некоторые сферы становятся особенно значимыми при депрессии, тогда как другие отступают «на задний план» (Бек и др., 2003)?

Субъективнье критерии оценки удовлетворенности жизнью $и$ счастья при непсихотических депрессиях и ремиссиях после первого психотического приступа

Мы предполагали, что, хотя основные сферы оценки качества жизни остаются значимыми для формирования общей удовлетворенности/неудовлетворенности при психических заболеваниях, относительный вклад этих сфер может меняться. В частности, при оценке удовлетворенности своей жизнью больные с депрессиями в большей степени опираются на свое отношение к своему здоровью, к функционированию в течение дня и эмоциональной сфере, 
тогда как для больных в ремиссии после психотического приступа, напротив, эмоциональная сфера и качество общения с окружающими менее важны. Кроме того, предполагалось, что субъективная значимость удовлетворенности (качества жизни) в разных сферах выше для удовлетворенности жизнью как результата когнитивной оценки (Diener, Ryan, 2009), нежели для субъективного счастья как основанного на эмоциональных переживаниях и менее дифференцированного (Аргайл, 2003).

Для проверки этих гипотез использовалась серия анализов модераций (Chaplin, 2007): при этом две зависимые переменные (удовлетворенность жизнью и субъективное счастье) предсказывались на основе (1) клинических особенностей группы (контрольная группа, больные с депрессиями, больные в ремиссии после приступа), (2) качества жизни в различных сферах (отдельно для каждой из сфер) и (3) взаимодействия между клиническими особенностями группы и качеством жизни в различных сферах. На этапе подготовки данных к анализу были созданы две бинарные переменные (так называемое простое кодирование dummy coding), описывающие три группы испытуемых: контрольной группе приписывались нули по обеим переменным (она выступала референтной группой, т.е. мы получали показатели относительно нее), больным с депрессиями - единицы по первой и нули по второй, больным в ремиссии - нули по первой и единицы по второй. Эти две переменные выступали в качестве независимых на первом шаге анализа модерации: при этом оценивалось, в какой степени удовлетворенность жизнью и счастье в нашей выборке объясняются наличием и типом диагноза. На втором шаге к ним добавлялась независимая переменная, характеризующая качество жизни в одной из конкретных сфер (например, в сфере здоровья). Значимое улучшение модели на данном этапе (увеличение процента объясненной дисперсии) означало, что качество жизни в данной сфере связано с общей удовлетворенностью жизнью или субъективным счастьем после контроля того/независимо от того, здоров ли человек, страдает депрессией или перенес психотический приступ. Наконец, для выявления собственно модерации (т.е. того, различается ли вклад качества жизни в конкретной сфере в общее благополучие в разных группах) две бинарные переменные групп испытуемых умножались на переменную качества жизни в каждой из сфер. Эти две новые переменные (модераторы) добавлялись в качестве независимых факторов на третьем шаге анализа модерации. Улучшение модели на третьем шаге означало, что вклад качества жизни в данной сфере в общую удовлетворенность жизнью или счастье различается в трех группах. Чтобы выяснить, в чем состоят эти различия, в случаях значимой модерации дополнительно рассчитывались простые регрессии (simple regression) отдельно в контрольной и двух клинических группах. Значимые положительные стандартизованные коэффициенты $\beta$ при этом свидетельствуют о наличии прямой связи, отрицательные - обратной связи между переменными. 
Вклад качества жизни в разных сферах в удовлетворенность жизнью и счастье после контроля различий между группами: результаты первого и второго шагов анализа модерации

Учет различий между клиническими и контрольной группами на первом шаге анализа модерации объясняет $14.1 \%$ дисперсии показателя удовлетворенности жизнью и $22.7 \%$ дисперсии показателя субъективного счастья в данном исследовании. Добавление в анализ показателей удовлетворенности конкретными сферами жизни на втором шаге анали- за модерации во всех случаях приводит к значимому улучшению модели, хотя и в разной степени (таблица 2).

Иными словами, во всех трех группах как удовлетворенность жизнью, так и субъективное счастье связаны с удовлетворенностью конкретными сферами жизни: здоровьем, эмоциями, общением, отношениями с другими людьми в целом, функционированием в течение дня. В меньшей степени удовлетворенность жизнью и субъективное счастье зависят от удовлетворенности активностью в свободное время, лекарствами (только в клинических

Таблица 2

Зависимость удовлетворенности жизныо и субъективного счастья от удовлетворенности конкретными сферами жизни после контроля различий между группами: результаты второго пага анализа модерации

\begin{tabular}{|l|c|c|c|c|}
\hline \multicolumn{1}{|c|}{$\begin{array}{c}\text { Субъективные критерии оценки } \\
\text { качества жизни }\end{array}$} & $\begin{array}{c}\text { Удовлетворенность своим } \\
\text { благополучием в целом }\end{array}$ & \multicolumn{2}{|c|}{$\begin{array}{c}\text { Субьективное } \\
\text { счасть }\end{array}$} \\
\hline & $\beta$ & $\triangle R^{2}$ Шаг 2 & $\beta$ & $\triangle R^{2}$ Шаг 2 \\
\hline Качество жизни в сфере здоровья & $0.41^{* *}$ & $15.3 \%$ & $0.35^{* *}$ & $11.3 \%$ \\
\hline Качество жизни в сфере эмоций & $0.69^{* *}$ & $37.4 \%$ & $0.55^{* *}$ & $24.2 \%$ \\
\hline $\begin{array}{l}\text { Качество жизни в сфере активности } \\
\text { в свободное время }\end{array}$ & $0.29^{* *}$ & $7.4 \%$ & $0.26^{* *}$ & $6.0 \%$ \\
\hline Качество жизни в сфере общения & $0.45^{* *}$ & $15.1 \%$ & $0.43^{* *}$ & $13.9 \%$ \\
\hline $\begin{array}{l}\text { Удовлетворенность лекарствами } \\
\text { (только в клинических группах) }\end{array}$ & $0.39^{* *}$ & $14.4 \%$ & $0.22^{* *}$ & $4.6 \%$ \\
\hline $\begin{array}{l}\text { Удовлетворенность своими } \\
\text { отношениями с другими людьми }\end{array}$ & $0.45^{* *}$ & $18.9 \%$ & $0.35^{* *}$ & $11.1 \%$ \\
\hline $\begin{array}{l}\text { Удовлетворенность } \\
\text { функцонированием в течение дня }\end{array}$ & $0.48^{* *}$ & $22.4 \%$ & $0.35^{* *}$ & $12.1 \%$ \\
\hline $\begin{array}{l}\text { Удовлетворенность своим } \\
\text { материальным благополучием }\end{array}$ & $0.38^{* *}$ & $14.4 \%$ & $0.17^{* *}$ & $2.9 \%$ \\
\hline
\end{tabular}

** $p<0.01$. Все $\triangle R^{2}$ значимы на уровне $p<0.01$. 
группах), а также своим материальным положением.

Эти результаты подтверждают вывод М. Ритснера с соавт. (Ritsner et al., 2005) о том, что в опроснике выделены сферы, значимые не только для здоровых людей, но и для пациентов с различными психическими заболеваниями.

Удовлетворенность лекарствами и материальным положением лишь слабо связана с эмоциональным переживанием субьективного счастья и в большей степени - с оценкой своей жизни и благополучия.

Особенности вклада качества жизни в разных сферах в удовлетворенность жизнью и счастье в контрольной группе при депрессиях и в ремиссиях после психотического приступа: результаты третьего шага анализа модерации

У больных с непсихотическими депрессиями качество жизни в сфере здоровья и удовлетворенность функционированием в течение дня более тесно связаны с удовлетворенностью жизнью $(\beta=0.33, p<0.01$ и $\beta=0.16$, $p<0.01$ соответственно), нежели в контрольной группе и при ремиссиях после психотического приступа (таблица 3). Та же закономерность характерна и для качества жизни в сфере эмоций, хотя в данном случае статистическая значимость объясняется не отличием больных с депрессиями от респондентов контрольной группы, а разницей между двумя клиническими группами: при депрессиях качество жизни в сфере эмоций более важно, а при ремиссиях - менее важно для удовлетворенности жизнью по сравнению с контрольной группой. Как видно по результатам простых регрессий (таблица 4), стандартизованный коэффициент, описывающий вклад качества жизни в сфере здоровья, эмоциональной сфере и удовлетворенность функционированием, в группе с депрессиями выше, чем в двух других группах. Кроме того, вклад удовлетворенности своим материальным благополучием в общую оценку удовлетворенности жизнью у больных в ремиссии после психотического приступа значимо ниже, чем в двух других группах $(\beta=-0.13, p<0.05)$.

Хотя качество жизни в эмоциональной сфере связано с субъективным счастьем во всех трех группах, эта связь слабее при непсихотических депрессиях $(\beta=-0.37, p<0.05)$ и особенно при ремиссиях после психотического приступа $(\beta=-0.62, p<0.01)$. Согласно простым регрессиям (таблица 4), стандартизованный коэффициент, описывающие вклад качества жизни в сфере эмоций в субъективное счастье, максимален в контрольной группе и минимален в группе с ремиссиями после психотического приступа. Качество жизни в сфере общения слабее связано с суб́ъективным счастьем в группе с ремиссиями после психотического приступа $(\beta=-0.43, p<0.05)$, хотя здесь модерация не достигает принятого уровня значимости $p<0.05$, оставаясь на уровне тенденции $p<0.07$. Удовлетворенность материальным благополучием связана с субъективным счастьем в контрольной группе, но не связана в группах с непсихотическими депрессиями и ремиссией после психотического приступа $(\beta=-0.13$, $p<0.05$ и $\beta=-0.16, p<0.05$ соответ ственно). 
Таблица 3

Особенности субъективных критериев оценки удовлетворенности жизнью при депрессиях и ремиссии после первого психотического приступа: результаты третьего шага анализа модерации

\begin{tabular}{|c|c|c|c|c|}
\hline \multirow{2}{*}{$\begin{array}{c}\text { Субъективные критерии } \\
\text { оценки качества жизни }\end{array}$} & \multicolumn{4}{|c|}{$\begin{array}{c}\text { Зависимая переменная: } \\
\text { Удовлетворенность своим благополучием в целом }\end{array}$} \\
\hline & $\begin{array}{l}\text { Контрольная } \\
\text { группа } \beta\end{array}$ & $\begin{array}{l}\text { Непсихотические } \\
\text { депрессии } \beta\end{array}$ & $\begin{array}{c}\text { Ремиссия после } \\
\text { психотического } \\
\text { приступа } \beta\end{array}$ & $\begin{array}{c}\triangle R^{2} \\
\text { Шаг } 3\end{array}$ \\
\hline $\begin{array}{l}\text { Качество жизни в сфере } \\
\text { здоровья }\end{array}$ & $0.34^{* *}$ & $0.63^{* *}$ & $0.32^{* *}$ & $2.0 \% *$ \\
\hline $\begin{array}{l}\text { Качество жизни в сфере } \\
\text { эмоций }\end{array}$ & $0.64^{* *}$ & $0.77^{* *}$ & $0.57^{* *}$ & $1.0 \% *$ \\
\hline $\begin{array}{l}\text { Удовлетворенность } \\
\text { функционированием в } \\
\text { течение дня }\end{array}$ & $0.45^{* *}$ & $0.65^{* *}$ & $0.51^{* *}$ & $1.7 \% * *$ \\
\hline $\begin{array}{l}\text { Удовлетворенность } \\
\text { своим материальным } \\
\text { благополучием }\end{array}$ & $0.51^{* *}$ & $0.43^{* *}$ & $0.24^{*}$ & $1.5 \% *$ \\
\hline
\end{tabular}

${ }^{*} p<0.05,{ }^{* *} p<0.01$.

Таблица 4

Особенности субъективных критериев оценки счастья при депрессиях и ремиссии после первого психотического приступа: результаты третьего шага анализа модерации

\begin{tabular}{|l|c|c|c|c|}
\hline \multirow{2}{*}{$\begin{array}{l}\text { Субъективные критерии } \\
\text { оценки качества жизни }\end{array}$} & \multicolumn{4}{|c|}{ Зависимая переменная: Субъективное счастье } \\
\cline { 2 - 5 } & $\begin{array}{c}\text { Контрольная } \\
\text { группа } \beta\end{array}$ & $\begin{array}{c}\text { Непсихотические } \\
\text { депрессии } \beta\end{array}$ & $\begin{array}{c}\text { Ремиссия после } \\
\text { психотического } \\
\text { приступа } \beta\end{array}$ & $\begin{array}{c}\triangle R^{2} \\
\text { Шаг } 3\end{array}$ \\
\hline $\begin{array}{l}\text { Качество жизни в сфере } \\
\text { эмоций }\end{array}$ & $0.64^{* *}$ & $0.58^{* *}$ & $0.47^{* *}$ & $2.2 \% * *$ \\
\hline $\begin{array}{l}\text { Качество жизни в сфере } \\
\text { общения }\end{array}$ & $0.48^{* *}$ & $0.45^{* *}$ & $0.33^{* *}$ & $1.2 \% \%^{\mathrm{T}}$ \\
\hline $\begin{array}{l}\text { Удовлетворенность своим } \\
\text { материальным } \\
\text { благополучием }\end{array}$ & $0.34^{* *}$ & 0.07 & 0.08 & $1.7 \% *$ \\
\hline
\end{tabular}

${ }^{\mathrm{T}} p<0.07$ (на уровне тенденции), ${ }^{*} p<0.05,{ }^{* *} p<0.01$. 


\section{Обсуждение результатов}

\author{
Возможности применения \\ русскоязычных версици Краткого \\ опросника качества жизни и \\ удовлетворенности и ШІкальь \\ счастья при психических \\ заболеваниях
}

Наши результаты подтверждают достаточную надежность-согласованность шкал опросника качества жизни и удовлетворенности и Шкалы счастья при непсихотических депрессиях и становлении ремиссий после психотического приступа. Кроме того, в соответствии с первой гипотезой, критериальная валидность опросника качества жизни и удовлетворенности подтверждается более низким уровнем качества жизни при непсихотических депрессиях по всем шкалам по сравнению с контрольной группой, что согласуется с данными других исследований (Nierenberg et al., 2010). У больных в становлении ремиссии после психотического приступа отмечается более низкий уровень качества жизни в сферах здоровья, эмоций и активности в свободное время, нежели в норме, однако их показатели выше, нежели при непсихотических депрессиях. Этот результат вполне согласуется как с данными о снижении критичности к своему состоянию у этих больных (Каплан, Сэдок, 2002), так и с особенностями их психопатологической симптоматики (меньшей выраженностью депрессивных симптомов). Конвергентная валидность подтверждается ожидаемым паттерном положительных корреляций шкал опросника с субъективным счастьем (в англоязычной версии эта закономерность была продемонстрирована для удовлетворенности жизнью - см.: Ritsner et al., 2005) и отрицательных - с целым рядом психопатологических симптомов (особенно это касается связей шкал качества жизни в сфере здоровья и эмоций со шкалами соматизации, обсессивности-компульсивности, межличностной тревоги и депрессии, характерных для обеих клинических групп).

Интересно, что при становлении ремиссии после психотического приступа обцая оценка удовлетворенности жизнью не только нередко не снижена по сравнению с нормой (различия не достигают принятого уровня значимости), но и не связана с выраженностью психопатологической симптоматики. Это же верно и для качества жизни в сфере общения. На наш взгляд, этот результат также свидетельствует в пользу валидности опросника, поскольку согласуется с данными о специфических мотивационных нарушениях при шизофрении (Критская и др., 1991; Зейгарник, 1986): обший вывод об удовлетворенности и о качестве своего общения носит иллюзорный и абстрактный характер, оставаясь «оторванным» от конкретного опыта больного. Следует отметить, что этот результат практически полностью повторяет полученный в англоязычном исследовании, где снижение качества жизни при шизофрении во многом определялось выраженностью нарушений регуляторных и когнитивных функций независимо от тяжести симптоматики (Ritsner, 2007).

Модификация опросника качества жизни и удовлетворенности для психических заболеваний (Ritsner et 
al., 2005) разрабатывалась таким образом, чтобы включать те сферы жизни, которые наиболее важны для пациентов. Результаты первых двух шагов анализа модераций подтверждают это предположение, свидетельствуя о содержательной валидности методики: все сферы, перечисленные в опроснике, были важны для общей оценки качества жизни не только для здоровых людей, но и для пациентов с различными психическими заболеваниями (хотя и в разной степени).

\section{Оценка удовлетворенности} жизнью и счастья как прочесс, опирающийся на оценку качества жизни в разньх сферах

В соответствии со второй и третьей гипотезами, во всех трех группах качество жизни по всем сферам связано с удовлетворенностью жизнью и счастьем, хотя выраженность этих связей варьирует. Иными словами, все перечисленные сферы в той или иной мере выступают в роли субъективных критериев при оценке своего благополучия. При этом качество жизни в сфере эмоций выступает в качестве центрального предиктора психологического благополучия, а качество жизни в сфере активности в свободное время связано с ним в минимальной степени.

В работах Э. Динера (Diener, Ryan, 2009) указывается, что психологическое благополучие определяется не только удовлетворенностью жизнью как результатом когнитивной оценки, но и эмоциональным состоянием. О неоднозначном статусе понятия суб́ьективного счастья как более холистической и эмоциональной оценке своего состояния также неоднократно говорилось в исследованиях (Lyubomirsky, 2013; Аргайл, 2003). Наши результаты также свидетельствуют в пользу того, что удовлетворенность и счастье различаются и с точки зрения процесса их оценки - того, на какие критерии опираются люди, принимая суждение о том, насколько они удовлетворены своей жизнью и насколько счастливы. Субъективное счастье в меньшей степени, чем удовлетворенность жизнью, зависит от оценок качества жизни в разных сферах; особенно это верно для сфер, требующих когнитивного анализа и сопоставления - функционирования в течение дня, материального благополучия, удовлетворенности лекарствами (процент объясняемой дисперсии для этих факторов почти в два и более раз выше для удовлетворенности, нежели для счастья). Таким образом, как на практике, так и в научных исследованиях диагностика удовлетворенности жизнью должна дополняться диагностикой других аспектов субъективного благополучия.

\section{Различия в субъективньх критериях оценки}

удовлетворенности жизнью $u$ счастья при депрессиях и ремиссиях после психотического приступа

Данное исследование основано на предположении, что оценка субъективного благополучия - это процесс, критерии и содержание которого могут быть изменены при психических заболеваниях. В подтверждение этой гипотезе было показано, что больные с непсихотическими депрессиями 
при оценке своей удовлетворенности жизнью (но не субъективного счастья) в большей степени, нежели респонденты контрольной группы и больные в ремиссии после психотического приступа, опираются на качество жизни в сфере здоровья, эмоций и функционирования в течение дня. В оценках субъективного счастья они, напротив, в несколько меньшей степени ориентируются на качество жизни в сфере эмоций и не ориентируются на материальное состояние по сравнению с нормой.

С позиций когнитивного подхода к депрессиям (Бек и др., 2003; Clark et al., 1999), негативные убеждения связаны к изб́рательным вниманием, при котором человек склонен замечать и интерпретировать события, поддерживающие эти убеждения, недооценивая альтернативные объяснения ситуации. В связи с этим ухудшение эмоционального состояния и здоровья не только вызывают большее беспокойство у больных эти сферы становятся особенно значимыми для них, определяя вывод об удовлетворенности жизнью в целом и за последнее время. Соответственно, особое значение приобретает функционирование в течение дня. Говоря метафорически, удовлетворенность жизнью начинает колебаться при депрессиях «в унисон» с событиями, происходящими в этих сферах. Следствием может являться так называемое «эмоциональное доказательство» (emotional reasoning) по типу «Если я расстроен, значит, мои опасения полностью верны» (Leahy, 2003). При этом другие, житейские моменты (например, материальное состояние) отходят на «второй План».
Отдельной интерпретации требуют противоположные эффекты модерации качества жизни в сфере эмоций в отношении удовлетворенности жизнью и счастья при депрессиях: эмоциональное состояние и здоровье становятся крайне важны для принятия когнитивного решения об удовлетворенности жизнью, но в несколько меньшей степени сказываются на холистическом, интуитивном переживании счастья. Немаловажно, что уровень счастья при депрессиях также снижен, но в меньшей степени, чем в норме, зависит от эмоциональной сферы. Хотя уточнение этого эффекта требует дальнейших исследований, мы предполагаем, что обобщенные и интуитивные оценки своего счастья при депрессиях являются скорее абстрактными, оторванными от реальных событий и переживаний в отличие от когнитивных оценок. Эта картина близка представлениям А. Бека о том, что при депрессиях затруднена или заблокирована сама возможности проверки выводов о себе, мире и своем будущем, исходя из текущих событий или динамики переживаний (Бек и др., 2003; Clark et al., 1999). Холистические выводы о своем счастье в большей степени определяются общими убеждениями, нежели состоянием в соответствующих жизненных сферах: образно говоря, убеждение «Я не доволен своей жизнью» включает для больного депрессией дополнение «потому что» (при этом особую значимость приобретают доказывающие это сферы), а убеждения «Я никогда не буду счастлив» или «Я не счастлив» принимаются как верные интуитивно, чувственные и не требующие оснований. 
В некотором смысле «восстановление» связи конкретных ситуаций, когнитивных и эмоциональных процессов и является задачей когнитивно-бихевиоральной терапии.

В отличие от больных с депрессиями у больных на этапе становления ремиссии после психотического приступа отмечаются одни и те же особенности оценки удовлетворенности жизнью и счастья: они в наименьшей степени ориентируются на эмоциональную сферу и материальное благополучие по сравнению с другими двумя группами респондентов. Кроме того, в оценках счастья они в меньшей степени ориентируются и на качество жизни в сфере общения. Можно предполагать, что значимость эмоциональных переживаний и общения снижается для этих больных, так же как и роль материального достатка. С одной стороны, эти результаты могут объясняться уже упоминавшимся мотивационным дефицитом и нарушениями в общении при шизофрении (Критская и др., 1991). При этом удовлетворенность жизнью и счастье могут выступать как «знаемые», будучи оторванными от реальной жизнедеятельности и опыта больного (Зейгарник, 1986). С другой стороны, снижение значимости эмоций, общения и материального положения в оценке благополучия может носить защитный характер, являющийся следствием реакции (в том числе избегания) на необычные переживания, нарастание негативной симптоматики и стигматизацию (Tarrier, 2010; Garety et al., 2001; Morrison, 2001). Косвенным подтверждением этого предположения является и тот факт, что при становлении ремиссии после психотического приступа общая удовлетворенность жизнью и качество жизни в сфере общения не связаны с психопатологическими симптомами, как бы «оторваны» от них.

\section{Заключение}

Таким образом, полученные данные свидетельствуют в пользу предположения о том, что процесс оценки своего благополучия в разной мере опирается на различные субъективные критерии (сферы). При этом, хотя общие критерии не различаются в норме и при психических заболеваниях, сам процесс оценки может быть искажен вследствие приписывания чрезмерной значимости одним критериям (сферам) и недооценке роли других критериев (сфер). С теоретической точки зрения это означает, что понимание определения, структуры и предикторов качества жизни и благополучия, доминировавшие в исследованиях качества жизни и благополучия до настоящего времени (см.: Рассказова, 2012a; Sirgy et al., 2006; Schalock, 2004), должно быть дополнено исследованиями процесса оценки своей жизни, его критериев и факторов как в норме, так и в патологии. Обращение к благополучию только как к зависимой переменной в исследованиях не дает возможности увидеть, что при различных заболеваниях речь может идти о по-разному сформированном представлении о своей жизни, для изменения которого требуются разные интервенции и которое может играть разную роль в жизни человека. Рассмотрение же оценки своей жизни как непрерывного динамического 
процесса, основанного на различной системе критериев и характеризующегося особой структурой в норме и патологии, открывает новые возможности для исследования качества жизни как источника и ресурса деятельности.

С практической точки зрения в соответствии с полученными данными любые попытки улучшения качества жизни и благополучия больных будут менее эффективны, если опираются на «обобщенное» представление о благополучии, не учитывая значимости разных сфер жизни в принятии решения о своем благополучии. Мишенью психотерапевтической работы при непсихотических депрессиях должно выступать не снижение качества жизни самого по себе, а те процессы принятия решения о качестве жизни, которые это снижение обуславливают (Бек и др., 2003; Clark et al., 1999): например, чрезмерное внимание к эмоциональному состоянию и здоровью. При становлении ремиссии после психо- тического приступа задача улучшения качества жизни должна включать задачу достижения соответствия между общим благополучием, качеством жизни в сфере общения и другими сферами жизни, а также психопатологической симптоматикой. Заметим, что эта задача шире, нежели формирование инсайта болезни (Тхостов и др., 2007), поскольку подразумевает работу с различными жизненными сферами больного, а также учет негативных симптомов и защитной реакции на них (Beck et al., 2009).

Интерес для дальнейших исследований представляет не только вопрос о процессе оценки жизни в норме и при психических заболеваниях, но и вопрос о регуляторных функциях этой оценки. Например, вызывает ли неудовлетворенность жизнью при депрессии усилия по ее изменению? Если вызывает, то какие и от каких факторов зависит эта регуляторная функция качества жизни и благополучия.

\section{Литература}

Аргайл, М. (2003). Психология сиастья. СПб.: Питер.

Бек, А., Раш, А., Шо, Б., Эмери, Г. (2003). Когиитивная терапия депрессии. СПб.: Питер.

Зейгарник, Б. В. (1986). Патопсихология. М.: Изд-во Московского университета.

Каплан, Г. И., Сэдок, Б. Дж. (2002). Клитиеская психиатрия (в 2 т.). М.: Медицина.

Осин, Е. Н., Леонтьев, Д. А. (2008). Апробация русскоязычных версий двух шкал экспресс-оценки субъективного благополучия. В кн. Материалы III Всероссийского социологического конгресса. М.: Ин-т социологии РАН/Российское общество социологов. Режим доступа: http://publications.hse.ru/chapters/78753840/

Критская, В. П., Мелешко, Т. К., Поляков, Ю. Ф. (1991). Патология психииеской деятельности при иизофрении: мотивация, общение, познание. М.: Изд-во Московского университета.

Рассказова, Е. И. (2012а). Качество жизни как междисциплинарная проблема: теоретические подходы и диагностика качества жизни в психологии, социологии и медицине. Теоретииеская и экспериментальная психология, 5(2), 59-71. 
Рассказова, Е. И. (2012б). Методика оценки качества жизни и удовлетворенности: психометрические характеристики русскоязычной версии. Психология. Журнал Высшей иколы экономи$\kappa и, 9(4), 81-90$.

Рассказова, Е. И., Тхостов, А. Ш. (2015). Биопсихосоциальный подход к пониманию здоровья и болезни. Обозрение психиатрии и медиииской психологии имени В.М. Бехтерева, 2, 17-21.

Садальская, Е. В., Ениколопов, С. Н. (2001). Психологические аспекты оценки качества жизни больных психосоматическими расстройствами. В кн. Психосоциальная реабилитация и КЖ: Сбортик науилых трудов (т. 137, с. 321-334). СПб.: Санкт-Петербургский научно-исследовательский психоневрологический институт им. В.М. Бехтерева.

Тарабрина, Н. В. (2001). Практикум по психологии посттравматического стресса. СПб.: Питер. Тхостов, А. Ш., Иржевская, В. П., Рупчев, Г. Е., Морозова, М. А. (2007). Инсайт в патопсихологии (исторические, теоретимеские и методологиеские аспекты). М.: Изд-во Московского университета.

Ссылки на зарубежные источники см. в разделе References после аналоязыиного блока.
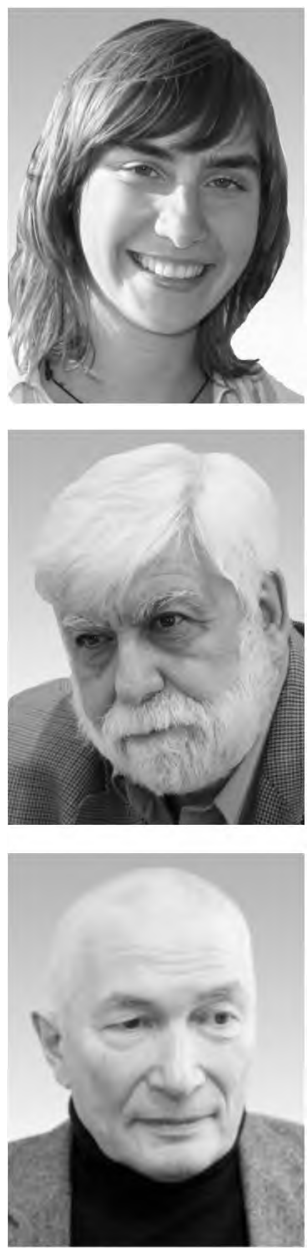

Рассказова Елена Игоревна - доцент, кафедра нейро- и патопсихологии, Московский государственный университет имени М.В. Ломоносова; старший научный сотрудник, лаборатория медицинской психологии ФГБНУ «Научный центр психического здоровья»; ведущий научный сотрудник, лаборатория позитивной психологии личности и мотивации, Национальный исследовательский университет «Высшая школа экономики», кандидат психологических наук. Сфера научных интересов: психология саморегуляции, психология здоровья, нарушения саморегуляции при психических и соматических заболеваниях, методы количественной обработки данных.

Контакты: e.i.rasskazova@gmail.com

Ениколопов Сергей Николаевич - профессор, факультет психологии, Московский государственный университет имени М.В. Ломоносова; заведующий отделом медицинской психологии, ФБГНУ «Научный центр психического здоровья», кандидат психологических наук.

Сфера научных интересов: клиническая психология, юридическая психология, криминальная психология, психология юмора. Контакты: enikolopov@mail.ru

Гульдан Виктор Викторович - руководитель психологической лаборатории Московского областного центра социальной и судебной психиатрии, доктор психологических наук, профессор. Сфера научных интересов: медицинская психология, судебная психология, криминология, общая и судебная психиатрия.

Контакты: vguldan@mail.ru 


\title{
Appraisal of Psychological Well-Being as Decision Making Process: Specificity of Subjective Criteria in Mental Illness (in Non-Psychotic Depression and a First Psychotic Episode in Youth)
}

\author{
Elena I. Rasskazova ${ }^{a, b, c}$, Sergey N. Enikolopov ${ }^{a, b}$, Victor V. Guldan ${ }^{\mathrm{d}}$ \\ ${ }^{a}$ The Mental Health Research Center, 34 Kashirskoe Highway, Moscore, 115522, Russian Federation \\ ${ }^{b}$ Lomonosov Moscow State University, 1 Leninskie Gony, Moscow, 119991, Russian Federation \\ 'National Research University Higher School of Economics, 20 Myasnitskaya Str., Moscow, 101000, \\ Russian Federation \\ ${ }^{\alpha}$ Moscoze Region Center of Social and Forensic Psychiatry, 18 Marta Str., Moscore, 1127083, Russian \\ Federation
}

\begin{abstract}
Subjective appraisal of quality of life, satisfaction and happiness is considered as a result of the decision making process, which could have different subjective criteria both in normative sample and in mental illnesses. We use concepts "satisfaction with life", "happiness" and "quality of life" as concrete aspects of integrative "psychological well-being" developing in different approaches. Using Quality of Life and Enjoyment Questionnaire (version for mental illnesses, Ritsner et al., 2005) and Lyubomirsky's Happiness Scale we compared in moderation analysis the contribution of the quality of life in different domains to the general appraisal of life satisfaction and subjective happiness in the three groups of young men (17-28 years old): non-psychotic depressive patients $\left(\mathrm{n}_{1}=76\right)$, patients developing remission after the first psychotic episode $\left(\mathrm{n}_{2}=90\right)$ and respondents without mental illness $\left(\mathrm{n}_{3}=185\right)$. Although all domains are important in different degrees for the general appraisal of satisfaction and happiness, in non-psychotic depression health, emotional sphere and functioning during the day are more important to their lives, compared with the two other groups. Patients developing remission after psychotic episode are less oriented to the emotional and social domains as well as financial well-being than participants from other two groups. The results are discussed in terms of the cognitive approach to understanding and psychotherapy for mental illnesses with an emphasis on the importance of taking into account subjective criteria and process of appraisal of general well-being in patients.
\end{abstract}

Keywords: quality of life, satisfaction with life, subjective happiness, subjective criteria of well-being, non-psychotic depression, remission after a psychotic episode, youth.

\section{References}

Argyle, M. (2003). Psikhologiya schast'ya [The psychology of happiness]. Saint Petersburg: Piter. (Transl. of: Argyle, M. (1987). The psychology of happiness. London: Methuen).

Beck, A. T., Rector, N. A., Stolar, N., \& Grant, P. M. (2009). Schizophrenia: Cognitive theory, research and therapy. New York: Guilford Press. 
Beck, A. T., Rush, A. J., Shaw, B. F., \& Emery, G. (2003). Kognitivnaya terapiya depressii [Cognitive therapy of depression]. Saint Petersburg.: Piter. (Transl. of: Beck, A. T., Rush, A. J., Shaw, B. F., \& Emery, G. (1979). Cognitive therapy of depression. New York: Guilford Press).

Chand, P.K., Mattoo, S.K., Sharan, P. (2004). Quality of life and its correlates in patients with bipolar disoreder stabilized on lithium prophylaxis. Psychiatry and Clinical Neurosciences, 58, 311-318.

Chaplin, W. F. (2007). Moderator and mediator models in personality research. In R. W. Robins, R. C. Fraley, \& R. E. Krueger (Eds.), Handbook of research methods in personality psychology (pp. 602-632). New York: The Guilford Press.

Clark, D. A., Beck, A. T., \& Alford, B. A. (1999). Scientific foundation of cognitive theory and therapy of depression. New York: John Wiley \& Sons.

Diener, E., \& Ryan, K. (2009). Subjective well-being: a general overview. South African Journal of Psychology, 39, 391-406.

Garety, P. A., Kuipers, E., Fowler, D., Freeman, D., \& Bebbington, P. E. (2001). A cognitive model of the positive symptoms of psychosis. Psychological Medicine, 31, 189-195.

Goracci, A., Martinucci, M., Kaperoni, A., Fagiolini, A., Sbaragli, C., Corsi, E., \& Castrogiovanni, P. (2007). Quality of life and subthreshold obsessive-compulsive disorder. Acta Neuropsychiatrica, 19, 357-361.

Goracci, A., Martinucci, M., Scalcione, U., Fagiolini, A., \& Castrogiovanni, P. (2005). Quality of life and subthreshold affective symptoms. Quality of Life Research, 14, 905-909.

Hope, M.L., Page, A.C., Hooke, G.R. (2009). The value of adding the Quality of Life Enjoyment and Satisfaction Questionnaire to outcome assessment of psychiatric inpatients with mood and affective disorders. Quality of Life Research, 18, 647-655.

Ilic, I., Milic, I., \& Arandelovic, M. (2010). Assessment quality of life: current approaches. Acta Medica Medianae, 49(4), 52-60.

Kaplan, H. I., \& Sadock, B. J. (2002). Klinicheskaya psikhiatriya [The clinical psychiatry] (in 2 Vols.). Moscow: Meditsina. (Transl. of: Kaplan, H. I., \& Sadock, B. J. (1972). Synopsis of psychiatry (3rd ed.). Baltimore/Hong Kong/London/Sidney: Williams and Wilkins).

Kritskaya, V. P., Meleshko, T. K., \& Polyakov, Yu. F. (1991). Patologiya psikhicheskoi deyatel'nosti pri shizofrenii: motivatsiya, obshchenie, poznanie [The pathology of the psychic activity in schizophrenia: motivation, communication, cognition]. Moscow: Moscow University Press.

Leahy, R. L. (2003). Cognitive therapy techniques: a practitioner's guide. New York/London: The Guilford Press.

Lyubomirsky, S. (2013). The myths of happiness: What should make you happy, but doesn't, what shouldn't make you happy, but does. New York: Penguin Press.

Lyubomirsky S., \& Lepper H. (1999). A measure of subjective happiness: Preliminary reliability and construct validation. Social Indicators Research, 46(2), 137-155.

Martin, F., Camfield, L., Rodham, K., Kleimpt, P., \& Ruta, D. (2007). Twelve years' experience with the Patient Generated Index (PGI) of quality of life: a graded structured review. Quality of Life Research, 16, 705-715.

McDowel, J. (2006). Measuring health. A guide to rating scales and questionnairies. New York: Oxford University Press.

Morrison, A. P. (2001). The interpretation of intrusions in psychosis: An integrative cognitive approach to hallucinations and delusions. Behavioural and Cognitive Psychotherapy, 29, 257-276. 
Nierenberg, A. A., Rapaport, M. H., Schettler, P. J., Howland, R. H., Smith, J. A., Edwards, D., ... Mischoulon, D. (2010). Deficits in psychological well-being and quality of life in minor depression: implications for DSM-V. CNS Neuroscience \& Tharapeutics, 16, 208-216.

Osin, E. N., \& Leontiev, D. A. (2008). Aprobatsiya russkoyazychnykh versii dvukh shkal ekspressotsenki sub»ektivnogo blagopoluchiya [Approbation of the Russian versions of the two scales of express-assessment of subjective well-being]. In Materialy III Vserossiiskogo Sotsiologicheskogo Kongressa [Proceedings of the $3^{\text {rd }}$ All-Russian Sociological Congress]. Moscow: Institute of Sociology of the Russian Academy of Sciences / Russian Society of Sociologists. Retrieved from http://publications.hse.ru/en/chapters/78753840

Rasskazova, E. I. (2012b). Evaluation of quality of life enjoyment and satisfaction: psychometric properties of a Russian-language measure. Psychology. Journal of Higher School of Economics, 9(4), 81 90. (in Russian)

Rasskazova, E. I. (2012a). Quality of life as an interdisciplinary problem: Theoretical approaches and diagnostics of the quality of life in psychology, sociology and medicine. Theoretical and Experimental Psychology, 5(2), 59-71. (in Russian)

Rasskazova, E. I., \& Tkhostov, A. Sh. (2015). Biopsychosocial approach in health psychology. V.M. Bekhterev Review of Psychiatry and Medical Psychology, 2, 17-21. (in Russian)

Ritsner, M. S. (2007). Predicting quality of life impairment in chronic schizophrenia from cognitive variables. Quality of Life Research, 16, 929-937.

Ritsner, M., Kurs, R., Gibel, A., Ratner, Y., \& Endicott, J. (2005). Validity of an abbreviated Quality of Life Enjoyment and Satisfaction Questionnaire (Q-LES-Q-18) for schizophrenia, schizoaffective, and mood disorder patients. Quality of Life Research, 14(7), 1693-1703.

Sadalskaya, E. V., \& Enikolopov, S. N. (2001). Psikhologicheskie aspekty otsenki kachestva zhizni bol'nykh psikhosomaticheskimi rasstroistvami [Psychological aspects of assessment of quality of life in patients with psychosomatic disorders]. In Psikhosotsial'naya reabilitatsiya $i$ KZh: Sbomik nauchrykh trudov [Psychosocial rehabilitation and quality of life: Collection of research papers] (Vol. 137, pp. 321-334). Saint Petersburg: Saint Petersburg V.M. Bekhterev Psychoneurological Research Institute.

Schalok, R. L. (2004). The concept of quality of life: what we know and do not know. Journal of Intellectual Disability Research, 48(3), 203-216.

Sirgy, M. J., Michalos, A. C., Ferris, A. L., Easterlin, R. A., Patrick, D., \& Pavot, W. (2006). The quality of life (QOL) research movement: past, present and future. Social Indicators Research, 76, 343-466.

Tarabrina, N. V. (2001). Praktikum po psikhologii posttravmaticheskogo stressa [A tutorial for the psychology of posttraumatic stress]. Saint Petersburg: Piter.

Tarrier, N. (2010). Cognitive behavior therapy for schizophrenia and psychosis: Current status and future directions. Clinical Schizophrenia and Related Psychoses, 4(3), 176-184.

Tkhostov, A. Sh., Irzhevskaya, V. P., Rupchev, G. E., \& Morozova, M. A. (2007). Insait v patopsikhologii (istoricheskie, teoreticheskie i metodologicheskie aspekty) [Insight in pathopsychology (historical, theoretical and methodological aspects)]. Moscow: Moscow University Press.

Wettergren, L., Kettis-Lindblad, A., Sprangers, M., \& Ring, L. (2009). The use, feasibility and psychometric properties of an individualised quality of life instrument: a systematic review of the SEIQoL-DW. Quality of Life Research, 18, 737-746.

Zeigarnik, B. V. (1986). Patopsikhologiya [Pathopsychology]. Moscow: Moscow University Press. 
Elena I. Rasskazova - associate professor, department of neuro- and pathopsychology, Lomonosov Moscow State University; senior researcher, laboratory of medical psychology, Mental Health Research Center; leading researcher, laboratory of positive psychology of personality and motivation, National Research University Higher School of Economics, Ph.D.

E-mail: e.i.rasskazova@gmail.com

Sergey N. Enikolopov - head of laboratory, laboratory of medical psychology, Mental Health Research Center; professor, department of psychology of personality, Lomonosov Moscow State University, Ph.D.

Research area: clinical psychology, forensic psychology, victimology, psychology of humor.

E-mail: enikolopov@mail.ru

Victor V. Guldan - head of the psychological laboratory, Moscow Region Center of Social and Forensic Psychiatry, D.Sc., professor.

Research area: clinical psychology, forensic psychology, criminology, general and forensic psychiatry.

E-mail: vguldan@mail.ru 